\title{
Insight into the mechanisms and consequences of recurrent telomere capture associated with a sub-telomeric deletion
}

\author{
Alexsandro dos Santos • Francine Campagnari - Ana Cristina Victorino Krepischi • \\ Maria de Lourdes Ribeiro Câmara • Rita de Cássia E. de Arruda Brasil • Ligia Vieira • \\ Angela M. Vianna-Morgante • Paulo A. Otto • Peter L. Pearson • Carla Rosenberg $\mathbb{D}$
}

Received: 19 February 2018 /Revised: 18 April 2018 / Accepted: 19 April 2018/Published online: 12 May 2018

(C) Springer Science+Business Media B.V., part of Springer Nature 2018

\begin{abstract}
A complex mosaicism of the short arm of chromosome 1 detected by SNP microarray analysis is described in a patient presenting a 4-Mb 1 p36 terminal deletion and associated phenotypic features. The array pattern of chromosome $1 \mathrm{p}$ displayed an intriguing increase in divergence of the SNP heterozygote frequency from the expected $50 \%$ from the centromere towards the 1p36 breakpoint. This suggests that various overlapping segments of UPD were derived by somatic recombination between the $1 \mathrm{p}$ homologues. The most likely explanation was the occurrence of a series of events initiated in either a gamete or an early embryonic cell division involving a 1 pter deletion rapidly followed by multiple telomere
\end{abstract}

Responsible Editor: Fengtang Yang.

Electronic supplementary material The online version of this article (https://doi.org/10.1007/s10577-018-9578-z) contains supplementary material, which is available to authorized users.

A. dos Santos · F. Campagnari · A. C. V. Krepischi •

L. Vieira · A. M. Vianna-Morgante · P. A. Otto •

P. L. Pearson $\cdot$ C. Rosenberg $(\square)$

Department of Genetics and Evolutionary Biology, Institute of

Biosciences, University of São Paulo, Rua do Matão 277, Sao

Paulo, SP 05508-090, Brazil

e-mail: carlarosenberg@uol.com.br

M. d. Ribeiro Câmara • R. d. C. E. de Arruda Brasil

Center of Odontological Assistance to Patients with Special

Needs, Faculty of Odontology, São Paulo State University,

Rodovia Marechal Rondon km 528, Aracatuba, SP 16018-395,

Brazil captures, resulting in additive, stepped increases in frequency of homozygosity towards the telomere. The largest segment involved the entire $1 \mathrm{p}$, and at least four other capture events were observed, indicating that at least five independent telomere captures occurred in separate cell lineages. The determination of breakpoint position by detection of abrupt changes in B-allele frequency using a moving window analysis demonstrated that they were identical in blood and saliva, the tissues available for analysis. We developed a model to explain the interaction of parameters determining the mosaic clones and concluded that, while number, size, and position of telomere captures were important initiating determinants, variation in individual clone frequencies was the main contributor to mosaic differences between tissues. All previous reports of telomere capture have been restricted to single events. Other cases involving multiple telomere capture probably exist but require investigation by SNP microarrays for their detection.

Keywords telomere capture $\cdot$ UPD $\cdot$ mosaicism $\cdot$ SNP microarray
Abbreviations
SNP Single nucleotide polymorphism
FISH Fluorescence in situ hybridization
$\mathrm{Mb} \quad$ Megabase
OMIM Online Mendelian Inheritance in Men
CNV Copy number variation
UPD Uniparental disomy
sUPD Segmental uniparental disomy 


\section{Introduction}

Monosomy 1p36 syndrome (OMIM \#607872) is caused by variably sized sub-telomeric deletions of the short arm of chromosome 1 (Shapira et al. 1997). Deletions of $1 \mathrm{p} 36$ are one of the commonest in humans and have an estimated incidence of 1:5000-1:10,000 (Heilstedt et al. 2003). Patients carrying the $1 \mathrm{p} 36$ deletion manifest cognitive impairment and characteristic facial features. They may also present other clinical features including seizures, growth and hearing impairment, hypotonia, and heart defects (Gajecka et al. 2007). Although the deletion size and breakpoint positions are variable between patients, the core clinical manifestations are fairly consistent (Shimada et al. 2014).

Four classes of chromosome rearrangements in individuals with monosomy $1 \mathrm{p} 36$ have been identified: derivatives of unbalanced translocations, interstitial deletions, apparently simple terminal truncations, and complex rearrangements. Simple terminal truncations, in which a sub-telomeric portion of $1 \mathrm{p} 36$ is lost along with the telomere, are the most common, and $67 \%$ of de novo rearrangements appear as simple terminal truncations at the sequence level (Gajecka et al. 2007; Heilstedt et al. 2003).

Eukaryotic chromosome stability relies on the presence of intact telomeres (Lustig 2003), and unless adequately repaired, telomere loss generates senescence and/or apoptotic cell death (Blasco 2005). Terminal deletions can be stabilized by "telomere healing," in which telomerase adds telomere sequences to the ends of the broken chromosomes, denominated "neotelomeres" (Chabchoub et al. 2007; Flint et al. 1994). Less frequently, terminal deletions can be stabilized by "telomere capture"(Bonaglia et al. 2011), with a terminally deleted chromosome acquiring a new telomere sequence usually from a chromatid of a normal homologue, and much less frequently from a heterologous chromosome. A common feature of telomere capture is that not only the telomere region itself is captured, but also variable lengths of the donor chromosome arm, effectively creating either segmental uniparental disomy (sUPD) of the chromosome arm, when captured from a homologue segment, or partial trisomy, when captured from a non-homologous segment. In such cases, the gain of a functional telomere presumably outweighs the disadvantages of creating allelic or genomic imbalances (Yu and Graf 2010). Additionally, but much less frequently, terminal deletions can also be stabilized by ring chromosome formation (Knijnenburg et al. 2007) or entering a breakage-fusion-bridge cycle (Ballif et al. 2003).

Here, we describe a terminal 1 p36 deletion associated with mosaic segmental uniparental disomy of the non-deleted part of $1 \mathrm{p}$. This was detected by SNP microarray analysis in a patient with clinical features of 1 p36 deletion syndrome. The heterozygosity frequency shows that the contribution of the twoparental chromosome 1 homologues increasingly deviates from $50 \%$, from the pericentromeric $1 \mathrm{p} 12$ region towards the $1 \mathrm{p} 36$ deletion breakpoint, compatible with mosaicism involving increasing levels of sUPD of different sized segments of $1 p$.

\section{Patients and methods}

Patient

The patient is a 26-year-old male born to healthy and non-consanguineous parents. The pregnancy was uncomplicated and delivered by cesarean section. At his birth, the father and mother were 21 and 20 years old, respectively. His older brother is healthy, and his mother had a spontaneous abortion at approximately 4 weeks' gestational age. His parents report a firstdegree cousin presenting severe intellectual disability. The patient presented a combination of developmental delay, intellectual disability, seizures, and delayed speech, compatible with features of the 1p36 deletion syndrome. His G-banded karyotype was normal.

Written informed consent for publication was obtained from the parents of the Patient. This research was approved by the Ethics Committee of the Biosciences Institute, University of São Paulo.

Saliva and peripheral blood samples from the patient and peripheral blood samples from both parents were obtained for molecular and cytogenetic studies.

We are willing to make DNA samples available from both blood and saliva from our patient to other groups wishing to collaborate on the molecular analysis of repetitive telomere capture phenomena. In this endeavor, the presence of four breakpoints associated with telomere capture within a single chromosome arm, combined with knowledge of their approximate location, should greatly assist in defining which types of DNA signature are most likely to be involved in telomere capture. 
SNP microarray

DNA was isolated using a standard phenol-chloroform protocol. Patient's genomic DNA both from saliva and blood were hybridized, according to the supplier's instructions, to a CytoSNP $850 \mathrm{~K}$ BeadChip (Illumina, USA), containing 850,000 SNP probes covering the whole-genome. Data were analyzed using BlueFuse Multi 4.1 Software (BlueGnome Ltd., Cambridge, UK), and log R ratio and $\mathrm{B}$-allele frequency (BAF) values were plotted along the chromosomal coordinates.

For the statistical analysis (Conover 1999), we used all microarray data points exported by the Bluefuse software. Only data for heterozygote genotypes were used, resulting in an average of $\sim 18 \times 10^{3}$ SNPS along the entire chromosome 1 or an average density of $\sim 73$ $\mathrm{SNPs}$ per $\mathrm{Mb}$. We developed a model predicting that Ballele variation along the short arm of chromosome 1 of this patient must occur in steps, in which the position of more abrupt and larger changes along the chromosome indicated the putative positions of capture events. Detection of breakpoint positions along $1 \mathrm{p}$ was performed using a moving window analysis in which statistically significant changes in B-allele frequency were detected by comparing the averages of the allele frequencies between consecutive $2 \mathrm{Mb}$ windows moved in $0.5 \mathrm{Mb}$ steps. The breakpoint positions were most easily recognized in saliva because of the generally higher amplitude of $\mathrm{B}$-allele changes compared to blood. However, the breakpoint positions established in blood closely matched those found in saliva to within several megabases but required a larger number of window movements to do so. The breakpoint locations were used in all further comparisons between tissues and segments; we applied Kruskal-Wallis non-parametric statistical analyses to evaluate changes in B-allele frequencies along the chromosome to determine UPD segment lengths and confirm breakpoint positions (Supplementary Material).

\section{FISH}

Metaphase chromosome spread preparations were obtained from lymphocyte cultures of peripheral blood, according to standard protocols. Fluorescence in situ hybridization (FISH) was performed on chromosome preparations from the patient and both parents. BAC probe RP11-465B22, which maps within the 1p36 segment deleted in the Patient, was labeled with biotin
(FITC; green), and a chromosome 1q44 probe (CTB$160 \mathrm{H} 23)$, used as a control, was labeled with digoxigenin (Rodamine; red).

\section{Results}

The SNP array analysis in both blood and saliva of the Patient revealed a constitutive $\sim 4 \mathrm{Mb}$ terminal deletion of the short arm of chromosome 1 ( $\operatorname{arr}[\mathrm{GRCh} 37]$ 1p36.33p36.32 (82,154_4,111,187)×1) (Fig. 1). The B-allele frequency (BAF) plot indicated complete absence of heterozygosity within the deleted segment, as expected. However, the frequency of heterozygous genotypes in the non-deleted $1 \mathrm{p}$ portion increasingly diverged from the expected $50 \%$ proportion from the centromere towards the deletion breakpoint; this could be explained by stepped reduction of heterozygosity matched by increases in mosaic uniparental disomy of $1 \mathrm{p}$ segments. This mosaic pattern was observed in both saliva and blood (Fig. 2a, b), the only tissues available for investigation. Despite the higher frequency of sUPD in saliva, the two tissue profiles were qualitatively very similar, with increasing levels of sUPD distributed in at least five segments along the whole $1 \mathrm{p}$. These patterns are consistent with the presence of the same five cell lines in both saliva and blood, but with different levels of mosaicism.

Although we were unable to directly visualize a consistent difference between segments $\mathrm{A}$ and $\mathrm{B}$ in blood, statistical testing of all possible segment differences for B-allele frequencies resulted in detection of a small but highly significant difference; this established that the telomere capture, comprising the full short arm of chromosome 1 (segment B) initially only detected in saliva, was also present in blood. Figure $2 \mathrm{c}$ displays the quantitative estimates of average B-allele frequencies calculated for each segment in blood, saliva and five controls; the minor differences between control segments were largely non-significant (see Supplementary Material) and most likely caused by variation between samples and array experiments.

Visual inspection of Figs. 2a, b shows that the position of four of the five observed breakpoints fall into the distal half of $1 \mathrm{p}$, a region long known to contain $\mathrm{G} / \mathrm{C}$ enriched stretches (Costantini et al. 2006) and a lower frequency of SNPs than in proximal $1 p$ (Hinds et al. 2006). Significantly, the most proximal breakpoint directly adjacent to the heterochromatic block associated 

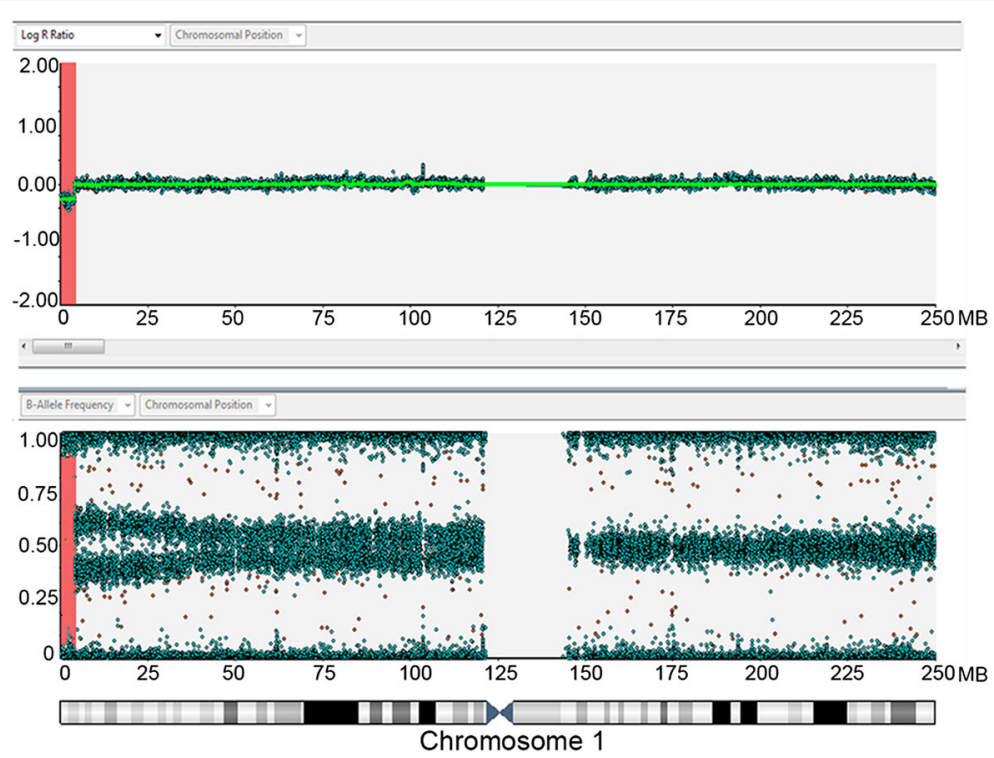

Fig. 1 SNP array analysis of chromosome 1 (saliva). Copy number profile (LogR ratio) is shown in the top panel and genotype (Ballele frequency) in the bottom panel. The copy number profile shows the $\sim 4 \mathrm{Mb}$ deletion (red bar) at $1 \mathrm{p} 36.33 \mathrm{p} 36.32$ (chr1:82,154_4,111,187;hg 19); as expected, the corresponding

with the centromere of chromosome 1 is also located in a region with localized $\mathrm{G} / \mathrm{C}$ enrichment. Figure $2 \mathrm{c}$ displays the quantitative estimates of average B-allele frequencies calculated for each segment in blood, saliva, and five controls. When examined at a higher resolution on the microarray intensity measurements, the five breakpoints were all located either within or directly adjacent to regions of a lower density SNP probe, which are indicated by the indentations on the outer edges of the SNP distribution in Figs. $2 \mathrm{a}$ and $\mathrm{b}$ at some of the breakpoint locations (the average SNP frequency of the 2-3 Mb surrounding and immediately adjacent to the five breakpoint locations averaged 39 per $\mathrm{Mb}$, in contrast to 96 per $\mathrm{Mb}$ in other regions of chromosome $1 \mathrm{p}$, a 2.5 -fold difference).

FISH on lymphocyte metaphases revealed the $1 \mathrm{p} 36$ deletion in most of the cells (94/100) (Fig. 3a). Although SNP microarray profiles did not show detectable heterozygosity in the $1 \mathrm{p} 36$ deletion region, signals of $1 \mathrm{pter}$ on both chromosomes 1 were observed in 6/100 metaphases (Fig. 3b). Retrospective evaluation of the $1 \mathrm{p} 36$ deletion profile (Fig. 1) showed that the $-0.25 \log R$ ratio was compatible with mosaicism, since the nonmosaic deletion $\log \mathrm{R}$ ratios in our cohort are approximately -0.35 .

Metaphases from both parents had normal hybridization patterns, pointing to the patient's deletion having
SNP pattern shows absence of heterozygosity in the deleted segment, but an unique pattern of stepped increases in allele frequency was observed in the non-deleted portion of $1 \mathrm{p}$. The heterozygosity increasingly positively diverged from the expected $50 \%$, from the centromere towards the $1 \mathrm{p} 36$ breakpoint

arisen as either a de novo $1 \mathrm{p} 36$ deletion in a parental gamete or an early embryonic cell division.

\section{Discussion}

Although terminal deletions have been described for every human chromosome, the molecular mechanisms that generate and stabilize such deletions remain incompletely understood.

Here, we describe a de novo terminal deletion of the short arm of chromosome 1 combined with a complex sUPD, present in both blood and saliva, the only tissues available for investigation. Earlier studies suggested that telomere capture was the most probable mechanism for stabilizing terminal deletions (Slijepcevic and Bryant 1998). However, more recent papers propose that stabilization of terminal deletions most frequently occurs by addition of telomere sequences directly onto the broken ends, i.e., addition of telomere sequences by telomerase, referred to as chromosome healing (Bonaglia et al. 2011; Fortin et al. 2009).

The most striking feature of the present case was the laddered divergence of a split pattern of B-allele frequency from the centromere towards the deletion breakpoint in the heterozygosity data of the SNP arrays, without copy number changes (Fig. 1). Our statistical 
Fig. 2 Data from the SNP arrays show variable B-allele frequencies on the short arm and proximal long arm of chromosome 1, in saliva (a) and blood (b). The orange line represents the expected average $50 \%$ B-allele frequency for heterozygosity. The profiles exhibit five levels of UPD mosaicism (B-F), with no detectable UPD on segment A. Note that, despite the higher frequency of cells with segmental UPD in saliva than blood, the tissue profiles are very similar. c The calculated average B-allele frequencies in heterozygosity for segments $\mathrm{A}$ to $\mathrm{F}$ in blood and saliva, and five controls are shown

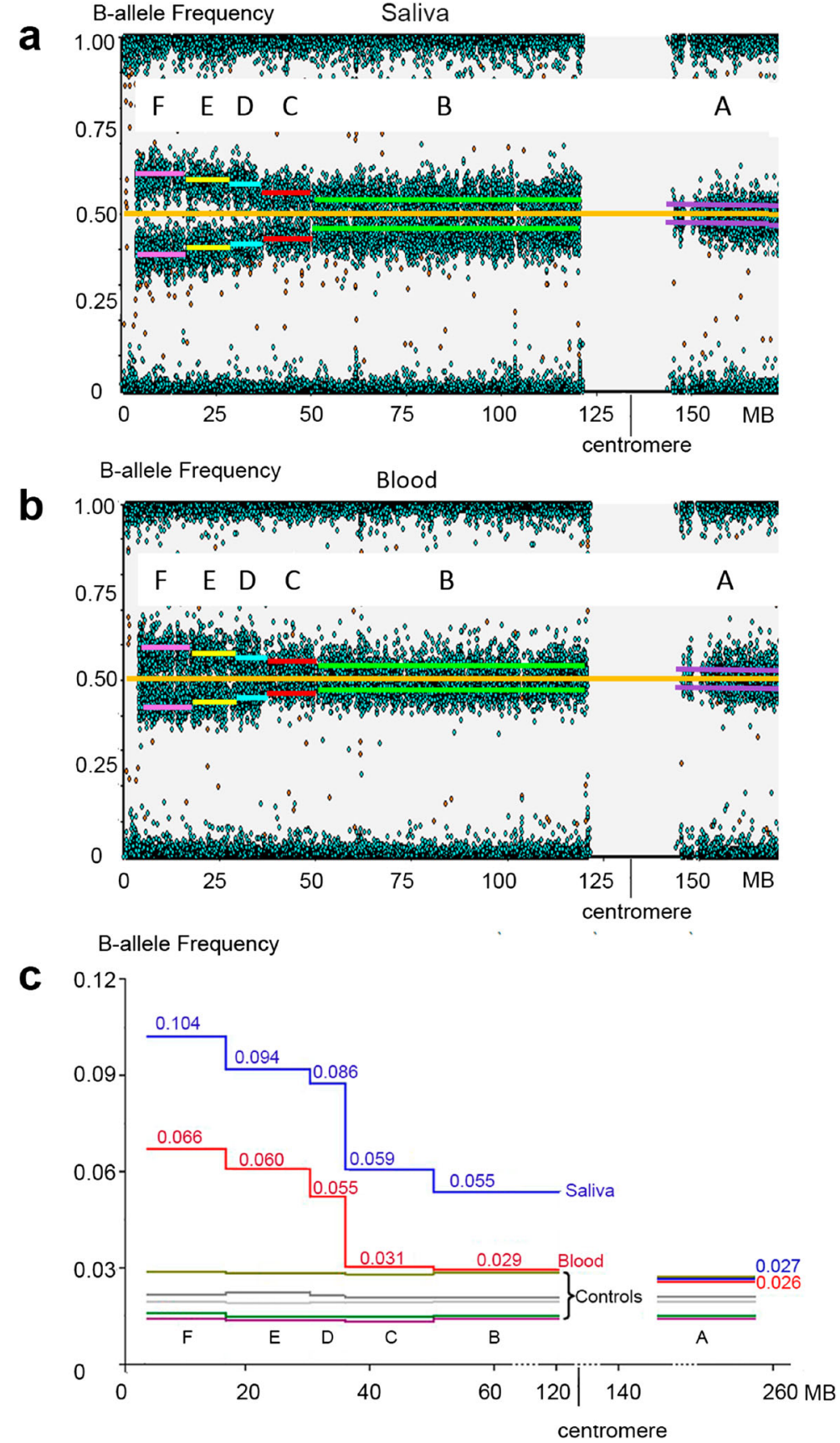

analyses show that this intriguing pattern can be explained by the additive overlap of B-allele frequencies within chromosome $1 p$ of five mosaic cell lines independently derived from the short arm of the normal chromosome 1 by somatic recombination through telomere capture, matched by a corresponding decrease in the proportion of the remaining $1 \mathrm{p}$ segments of the deleted chromosome 1 . The presence of five similar mosaic cell lines in both tissues implies that the independent telomere capture events must have all occurred in a precursor of both tissues, presumably at an extremely early stage of embryogenesis. A schematic representation of the events giving rise to this complex UPD mosaicism is given in Fig. 4. According to this model, the deletion detected by microarray analyses would be mosaic, with each type of mosaic cell line 
a

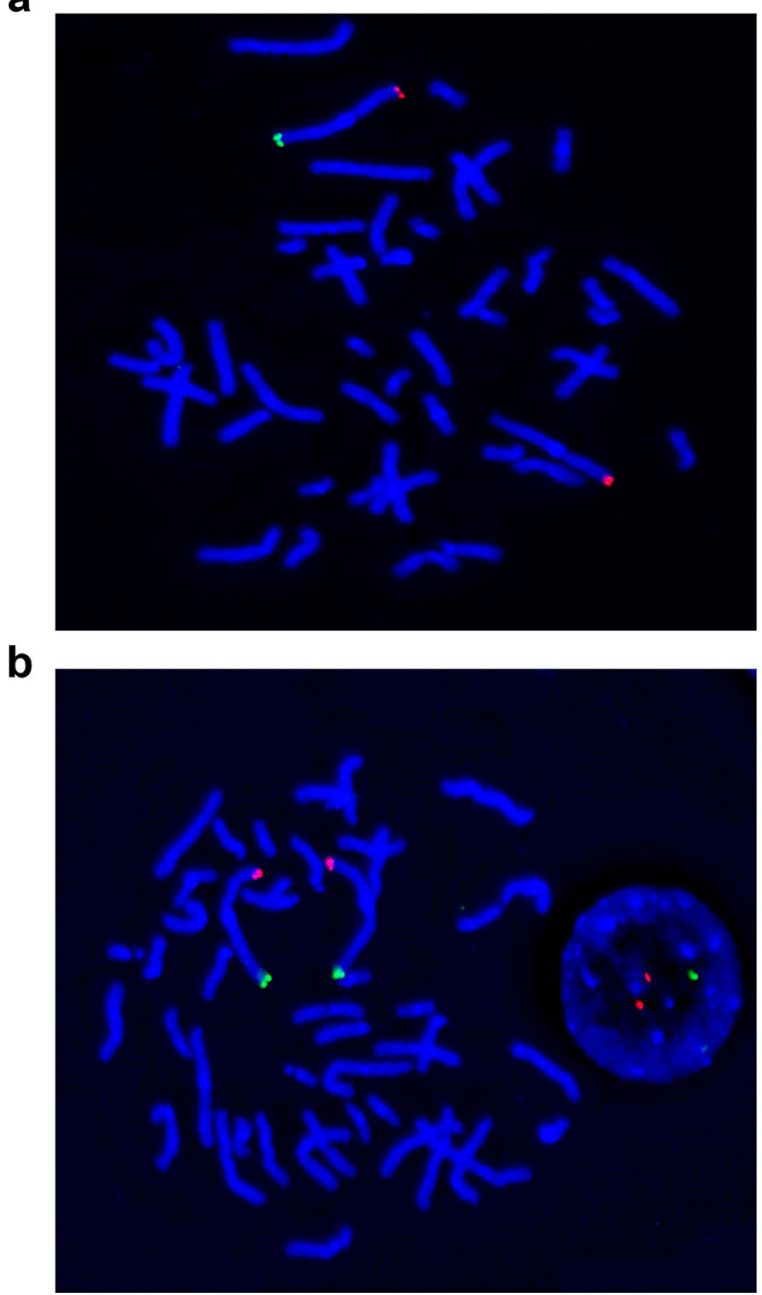

Fig. 3 FISH using a probe that maps to the 1 p36 segment deleted in the patient (green signal), and of a control probe located at the sub-telomeric region of the long arm of chromosome 1 (red signal), on cultured lymphocyte metaphases from the proband. a The green signal is observed on the short arm of only one chromosome 1, consistent with the $1 \mathrm{p} 36$ deletion detected by SNP array analyses, while the control red signal is observed on both chromosomes. b The green signal is observed on the short arm of both chromosomes 1 , showing the presence of a minor cell line without the 1 p36 deletion. The pictures were taken in a Photomicroscope Zeiss with a $\times 10$ ocular and $\times 100$ objective

containing different but partially overlapping stretches of $1 p$ UPD segments. Indeed, the FISH analysis showed that two patterns of hybridization were present: one without a sub-telomeric signal in one $1 \mathrm{p}$ homologue, confirming the original $1 \mathrm{p} 36$ deletion, and the other with $1 \mathrm{p} 36$ signals present on both homologues denoting occurrence of telomere capture (Fig. 3a, b). Regarding cells that had retained the $1 \mathrm{p} 36$ deletion, we assume that the deleted chromosome had become stabilized through creation of a neo-telomere by telomerase activity (telomere healing), although this was difficult to confirm in all metaphases due to the presumptive small size of the neo-telomere repeat regions (Sprung et al. 1999) and the impossibility of morphologically distinguishing the normal and deleted chromosome 1 homologues.

Although we have no independent method to infer how variations in B-allele frequency in our patient directly relate to the frequency of mosaicism of each cell line, the classic study of Conlin et al. (2010) on variations in B-allele frequency between independent patients, exhibiting various levels of mosaicism (Conlin et al. 2010), suggests that the $\mathrm{F}$ segment in saliva from our patient is present in $\sim 20 \%$ of the cells, decreasing to $\sim 5 \%$ in segment $\mathrm{B}$; the corresponding values in blood vary between $\sim 15$ and $\sim 2 \%$, all significantly lower than those observed in saliva. The main differences in allele profiles between blood and saliva are largely attributable to the higher frequency of cells with deletion of the entire $1 p$ (segment $B$ ) in saliva than in blood, resulting in a larger addition to the B-allele frequencies of the downstream segments $C$ to $F$. However, although the ratio of mosaicism between adjacent captured segments in both tissues remains approximately the same, it is not identical, probably due to genetic drift arising from differences in cell line proliferation during the differentiation process.

The breakpoints appear to have arisen in regions with a localized lower frequency of SNPs and a more broadly dispersed increase in G/C content. Several studies claim that such regions have an enhanced likelihood of repeat sequence mediated somatic recombination (Costantini and Bernardi 2009) similar to that more recently postulated for $\mathrm{CNV}$ initiation (Bose et al. 2014).

We have no firm idea which of the putative repetitive sequences are likely to be involved, and further molecular analyses comparing the DNA structure of the five breakpoints within the same chromosome arm will be required: this endeavor should be greatly assisted by the a priori information on the approximate breakpoint locations.

Although there are multiple descriptions of segmental UPD arising in cancer cells leading to mosaicism between different cell lines (Rumi et al. 2011; Makishima and Maciejewski 2011), there are, as yet, no documented instances of this occurring in cancer by telomere loss followed by subsequent telomere capture and its sequelae (for review, see Maciejowski and de 


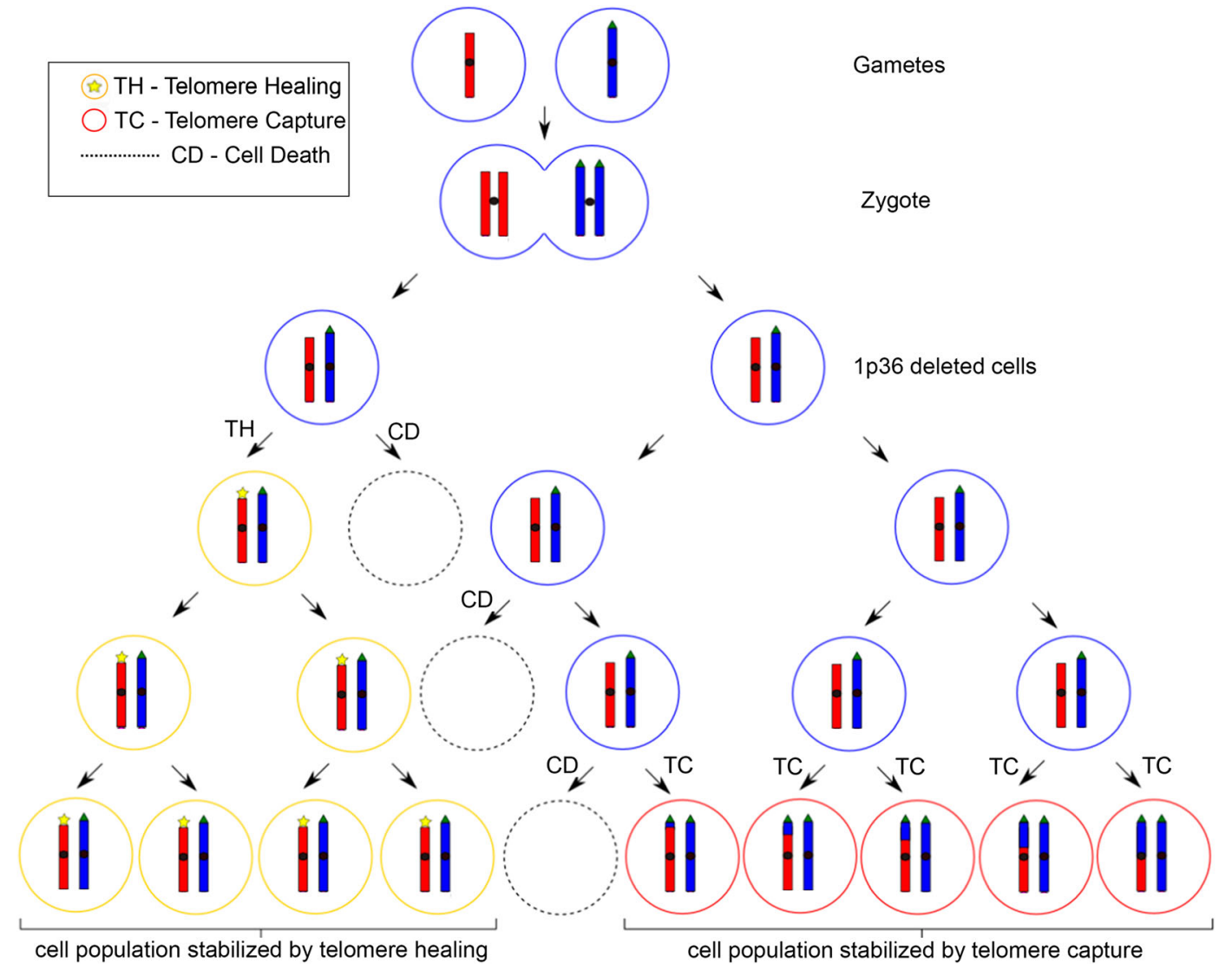

Fig. 4 A model for the origin of the complex mosaic 1p segmental uniparental disomy. A heterozygous $1 \mathrm{p} 36$ terminal deletion originated either in a gamete or early in the embryo. Unless the missing telomere is reinstated, cell death occurs. In an early division, telomere healing gave rise to a preponderant cell population with the original $1 \mathrm{p} 36$ deleted chromosome, but with addition of neotelomeres (telomere healing). In cells with an unrepaired $1 \mathrm{p} 36$

Lange 2017). This may be because, even if telomere loss and recapture does occur in cancer cells, it may be extremely difficult to distinguish from UPD arising through conventional chromatid recombination mechanisms, unless the original deleted chromosome remains as evidence in at least one of the derived cancer cell lines.

Martin et al. (2016) investigated a mosaic 20p13 $\rightarrow$ pter deletion by SNP array analysis and proposed rescue by mitotic recombination as the mechanism leading to one population of cells with a 20p deletion and another with normal copy number, but exhibiting segments of UPD (homozygosity) in 20 p $13 \rightarrow$ pter. However, multiple telomere captures arising from one particular rearrangement have never been documented previously, either because recurrent telomere capture is an extremely rare event or because, in the absence of deletion, recurrent telomere capture by mitotic homologous recombination produces various populations of cells with homozygosity of the $1 \mathrm{p}$ homologous telomere as well as variable lengths of the normal homologous short arm, dependent upon the position of recombination. The overlap of different lengths of segmental UPD in these cells explains the unusual SNP pattern observed by array analysis (Figs. 1 and 2)

genotype profiling derived from SNP arrays, it cannot be distinguished from a single telomere capture.

In conclusion, although in the present case telomere capture appears to be the primary mechanism responsible for stabilization of the deleted chromosome, the independent and repetitive nature of the captures resulted in transfer of multiple segments of various lengths from the normal $1 \mathrm{p}$ to the deleted $1 \mathrm{p}$. This, in combination with a cell population stabilized by telomere healing, gave rise to an extremely complex admixture of $1 p$ UPD in the two tissues studied.

Funding information This work was supported by scholarships from the Brazilian National Council for Scientific and Technological Development (CNPq-130185/2014-0; AS and 306879/2014-0; CR) and grants from the São Paulo Research Foundation (FAPESP_-2012/50981-5 and 2013/08028-1). 
Compliance with ethical standards Written informed consent for publication was obtained from the parents of the Patient. This research was approved by the Ethics Committee of the Biosciences Institute, University of São Paulo.

Competing interests The authors declare that they have no competing interests.

\section{References}

Ballif BC, Yu W, Shaw CA, Kashork CD, Shaffer LG (2003) Monosomy 1p36 breakpoint junctions suggest pre-meiotic breakage-fusion-bridge cycles are involved in generating terminal deletions. Hum Mol Genet 12:2153-2165. https://doi.org/10.1093/hmg/ddg231

Blasco MA (2005) Telomeres and human disease: ageing, cancer and beyond. Nat Rev Genet 6:611-622. https://doi. org/10.1038/nrg1656

Bonaglia MC, Giorda R, Beri S, de Agostini C, Novara F, Fichera M, Grillo L, Galesi O, Vetro A, Ciccone R, Bonati MT, Giglio S, Guerrini R, Osimani S, Marelli S, Zucca C, Grasso R, Borgatti R, Mani E, Motta C, Molteni M, Romano C, Greco D, Reitano S, Baroncini A, Lapi E, Cecconi A, Arrigo G, Patricelli MG, Pantaleoni C, D'Arrigo S, Riva D, Sciacca F, Dalla Bernardina B, Zoccante L, Darra F, Termine C, Maserati E, Bigoni S, Priolo E, Bottani A, Gimelli S, Bena F, Brusco A, di Gregorio E, Bagnasco I, Giussani U, Nitsch L, Politi P, Martinez-Frias ML, Martínez-Fernández ML, Martínez Guardia N, Bremer A, Anderlid BM, Zuffardi O (2011) Molecular mechanisms generating and stabilizing terminal 22q13 deletions in 44 subjects with Phelan/McDermid syndrome. PLoS Genet 7:e1002173. https://doi.org/10.1371/journal.pgen.1002173

Bose P, Hermetz KE, Conneely KN, Rudd MK (2014) Tandem repeats and G-rich sequences are enriched at human $\mathrm{CNV}$ breakpoints. PLoS One 9:e101607. https://doi.org/10.1371 /journal.pone.0101607

Chabchoub E, Rodriguez L, Galan E, Mansilla E, Luisa MartinezFernandez M, Luisa Martinez-Frias M, Fryns JP, Robert Vermeesch J (2007) Molecular characterisation of a mosaicism with a complex chromosome rearrangement: evidence for coincident chromosome healing by telomere capture and neo-telomere formation. J Med Genet 44:250-256. https://doi.org/10.1136/jmg.2006.045476

Conlin LK, Thiel BD, Bonnemann CG, Medne L, Ernst LM, Zackai EH, Deardorff MA, Krantz ID, Hakonarson H, Spinner NB (2010) Mechanisms of mosaicism, chimerism and uniparental disomy identified by single nucleotide polymorphism array analysis. Hum Mol Genet 19:1263-1275. https://doi.org/10.1093/hmg/ddq003

Conover WJ (1999) Practical non-parametric statistics, 3rd edn. Wiley, New York

Costantini M, Bernardi G (2009) Mapping insertions, deletions and SNPs on Venter's chromosomes. PLoS One 4:e5972. https://doi.org/10.1371/journal.pone.0005972

Costantini M, Clay O, Auletta F, Bernardi G (2006) An isochore map of human chromosomes. Genome Res 16:536-541

Flint J, Craddock CF, Villegas A, Bentley DP, Williams HJ, Galanello R, Cao A, Wood WG, Ayyub H, Higgs DR
(1994) Healing of broken human chromosomes by the addition of telomeric repeats. Am J Hum Genet 55:505-512

Fortin F, Beaulieu Bergeron M, Fetni R, Lemieux N (2009) Frequency of chromosome healing and interstitial telomeres in 40 cases of constitutional abnormalities. Cytogenet Genome Res 125:176-185. https://doi.org/10.1159/000230002

Gajecka M, Mackay KL, Shaffer LG (2007) Monosomy 1p36 deletion syndrome. Am J Med Genet C Semin Med Genet 145C:346-356. https://doi.org/10.1002/ajmg.c.30154

Heilstedt HA, Ballif BC, Howard LA, Kashork CD, Shaffer LG (2003) Population data suggest that deletions of $1 \mathrm{p} 36$ are a relatively common chromosome abnormality. Clin Genet 64 : 310-316

Hinds DA, Kloek AP, Jen M, Chen X, Frazer KA (2006) Common deletions and SNPs are in linkage disequilibrium in the human genome. Nat Genet 38:82-85

Knijnenburg J, van Haeringen A, Hansson KBM, Lankester A, Smit MJM, Belfroid RDM, Bakker E, Rosenberg C, Tanke HJ, Szuhai K (2007) Ring chromosome formation as a novel escape mechanism in patients with inverted duplication and terminal deletion. Eur J Hum Genet 15:548-555

Lustig AJ (2003) Clues to catastrophic telomere loss in mammals from yeast telomere rapid deletion. Nat Rev Genet 4:916923. https://doi.org/10.1038/nrg1207

Maciejowski J, de Lange T (2017) Telomeres in cancer: tumor suppression and genome stability. Nat Rev Mol Cell Biol 18: 175-186. https://doi.org/10.1038/nrm.2016.171

Makishima H, Maciejewski J (2011) Pathogenesis and consequences of uniparental disomy in cancer. Clin Cancer Res 17:39133923. https://doi.org/10.1158/1078-0432.CCR-10-2900

Martin MM, Vanzo RJ, Sdano MR, Baxter AL, South ST (2016) Mosaic deletion of 20pter due to rescue by somatic recombination. Am J Med Genet A 170A(1):243-248. https://doi. org/10.1002/ajmg.a.37407

Rumi E, Harutyunyan A, Elena C, Pietra D, Klampfl T, Bagienski K, Berg T, Casetti I, Pascutto C, Passamonti F, Kralovics R, Cazzola M (2011) Identification of genomic aberrations associated with disease transformation by means of high-resolution SNP array analysis in patients with myeloproliferative neoplasm. Am J Hemat 86:974-979. https://doi. org/10.1002/ajh.22166

Shapira SK, McCaskill C, Northrup H, Spikes AS, Elder FFB, Sutton VR, Korenberg JR, Greenberg F, Shaffer LG (1997) Chromosome 1p36 deletions: the clinical phenotype and molecular characterization of a common newly delineated syndrome. Am J Hum Genet 61:642-650. https://doi.org/10.1086/515520

Shimada S, Maegaki Y, Osawa M, Yamamoto T (2014) Mild developmental delay and obesity in two patients with mosaic 1p36 deletion syndrome. Am J Med Genet A 164A:415-420. https://doi.org/10.1002/ajmg.a.36304

Slijepcevic P, Bryant PE (1998) Chromosome healing, telomere capture and mechanisms of radiation-induced chromosome breakage. Int J Radiat Biol 73:1-13

Sprung CN, Reynolds GE, Jasin M, Murnane JP (1999) Chromosome healing in mouse embryonic stem cells. Proc Natl Acad Sci U S A 96:6781-6786

Yu S, Graf WD (2010) Telomere capture as a frequent mechanism for stabilization of the terminal chromosomal deletion associated with inverted duplication. Cytogenet Genome Res 129:265-274. https://doi.org/10.1159/000315887 